\title{
Detection of emerging rotavirus G12P[8] in Sonora, México
}

\author{
G. GONZÁLEZ-OCHOA', G. DE J. QUINTERO-OCHOA', P. M. CALLEJA-GARCÍA', J. A. ROSAS-RODRÍGUEZ1', \\ A. VIRGEN-ORTÍZ' ${ }^{2}$, P. TAMEZ-GUERRA ${ }^{3}$
}

\begin{abstract}
${ }^{1}$ Departamento de Ciencias Químico Biológicas y Agropecuarias, Universidad de Sonora, Sonora, México; ${ }^{2}$ Centro Universitario de Investigaciones Biomédicas, Universidad de Colima, Colima, México; ${ }^{3}$ Laboratorio de Inmunología y Virología, Universidad Autónoma de Nuevo León, Nuevo León, México
\end{abstract}

Received April 21, 2015; revised October 12, 2015; accepted April 15, 2016

\begin{abstract}
Summary. - Rotavirus is the most common cause of gastroenteritis in children up to five years of age worldwide. The aim of the present study was to analyze the genotypes of rotavirus strains isolated from children with gastroenteritis, after the introduction of the rotavirus vaccine in México. Rotavirus was detected in 14/100 (14\%) fecal samples from children with gastroenteritis, using a commercial test kit. The viral genome was purified from these samples and used as a template in RT-PCR amplification of the VP4 and VP7 genes, followed by gene cloning and sequencing. Among the rotavirus strains, 4/14 (28.5\%) were characterized as G12P[8], 2/14 (14.3\%), as G12P (not typed), and 3/14 (21.42\%) as G (not typed) P[8]. Phylogenetic analysis of the VP7 gene showed that G12 genotypes clustered in lineage III. Phylogenetic analysis revealed that VP4 genotype P[8] sequences clustered in lineage V, whereas other $\mathrm{P}[8]$ sequences previously reported in Mexico (2005-2008) clustered in different lineages. Rotavirus genotype G12 is currently recognized as a globally emerging rotavirus. To our knowledge, this is the first report of this emerging rotavirus strain G12P[8] in México. Ongoing surveillance is recommended to monitor the distribution of rotavirus genotypes and to continually reassess the suitability of currently available rotavirus vaccines.
\end{abstract}

Keywords: rotavirus; gastroenteritis; G12P[8]

\section{Introduction}

Rotavirus gastroenteritis is a common infectious disease that affects children up to five years of age worldwide (Desselberger et al., 2009). The mortality rates from diarrhea attributable to rotavirus infection are estimated to be 453,000 child deaths per year, with most of the deaths occurring in developing countries (Tate et al., 2012). Rotavirus genotypes G1P[8], G2P[4], G3P[8], G4P[8], and G9P [8] are responsible for the majority of severe cases of infections worldwide and currently available rotavirus vaccines were designed based on these five common circulating genotypes (Parashar et al., 2013). Despite the worldwide prevalence of rotavirus genotypes G1-G4 and G9, several

E-mail: lupitaglezo@hotmail.com; phone: +526424259969. studies have described unusual genotypes such as G5, G8, and G12 (Rahman et al., 2007; Santos and Hoshino, 2005). The first isolate of G12 rotavirus was reported in 1987, causing diarrhea in young children. It was not reported again until 1998 in Thailand, then subsequently in 1999-2006 in United States, India, Bangladesh, Japan, Korea, United Kingdom, Belgium, Argentina, and Brazil (Rahman et al., 2007). G12 is currently recognized as a globally emerging rotavirus genotype, displaying a high rate of transmission in recent years (da Silva Soares et al., 2012; Delogu et al., 2015; Tort et al., 2015). In 2006, the Rotavirus vaccine program was implemented in México. Although the currently available vaccine has been described as safe and efficacious, the variability in rotavirus genotypes over recent years highlights the need for constant surveillance. Therefore, the present study reports genotypic analysis of rotavirus strains isolated from children with gastroenteritis in the south of Sonora, México. 


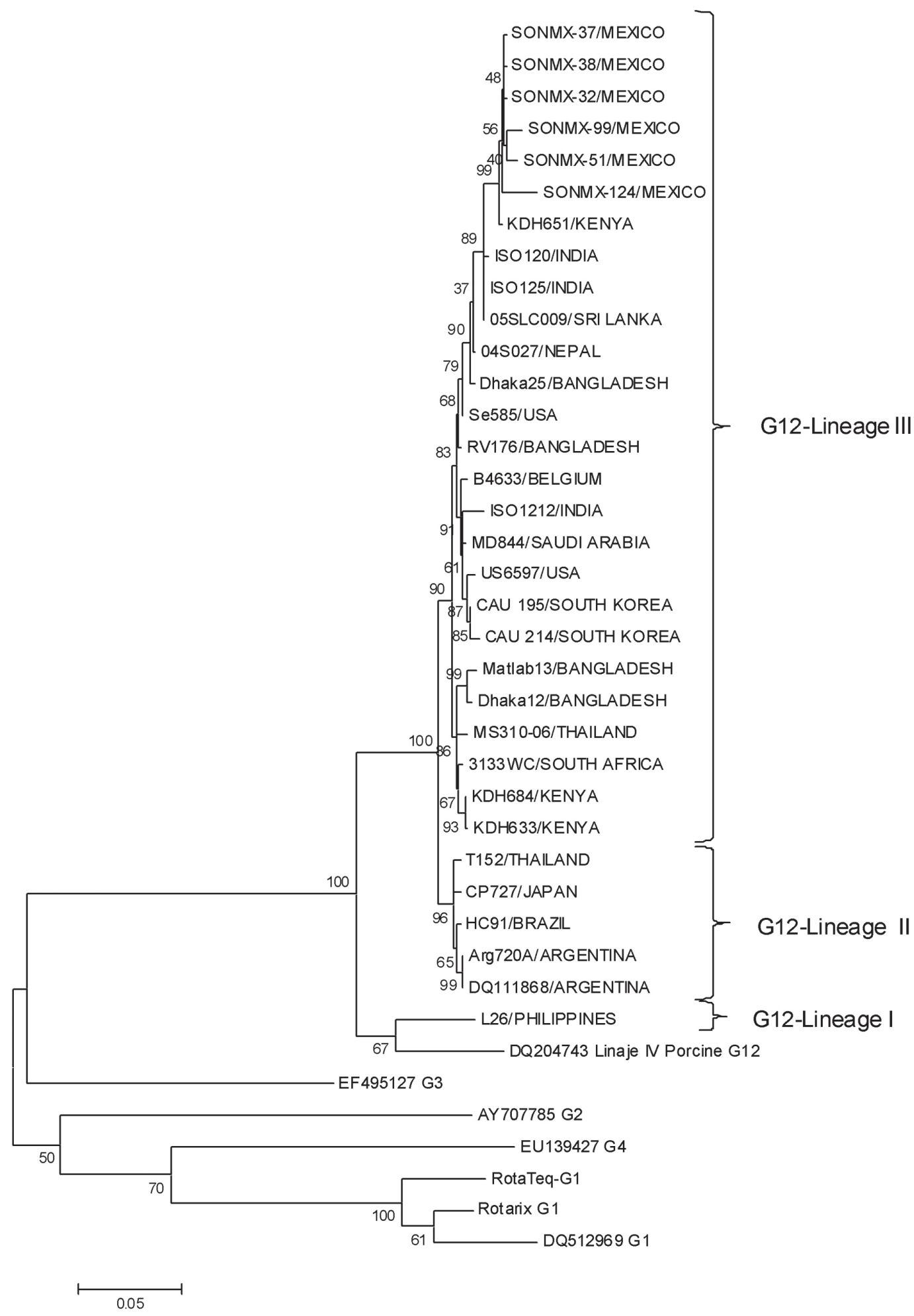

Fig. 1

Phylogenetic analysis of the VP7 gene of genotype G12 rotavirus

Phylogenetic analysis of the nucleotide sequence of the VP7 gene of genotype G12 rotavirus strains reported in this study (SONMX-32, SONMX-37, SONMX-38, SONMX-62, SONMX-99, SONMX-124) along with reference strains downloaded from the GenBank database. The phylogenetic relationships between strains were reconstructed using the maximum likelihood method. The statistical significance of the phylogenetic trees constructed was estimated by the bootstrap method (1000 replicates). 


\section{Material and Methods}

Sample collection. A total of 100 stool samples were collected from children with gastroenteritis, between August 2012 and August 2013, at Hospital del Niño y la Mujer in Cd. Obregón and Hospital General in Navojoa; both hospitals are located south of Sonora, México. To detect rotavirus positive samples, a rapid test for the detection of rotavirus antigen in stools was used according to the manufacturer's instructions (One Step Rota Adenovirus Antigen Test, Standard Diagnostics, Inc., Republic of Korea).

DNA extraction and cDNA cloning. From the rotavirus positive fecal samples, double-stranded RNA (dsRNA) was extracted using the Trizol ${ }^{\circledR}$ method (Life Technologies, USA). The purified dsRNA was used as a template for RT-PCR to amplify the VP4 (genotype P) and VP7 (genotype G) gene sequences, as previously described (Gouvea et al., 1990; Gentsch et al., 1992; Das et al., 1994; Simmonds et al., 2008). The PCR products of the VP4 and VP7 amplified genes were cloned into the pGEM-T vector (Promega, USA), following the manufacturer's instructions. Plasmids containing the VP4 or VP7 insert were purified using the Wizard SV Miniprep kit (Promega) and sequenced by the dideoxynucleotide chain termination method, using an ABI Prism BigDye terminator cycle sequencing ready reaction kit (Applied Biosystems, USA). The DNA sequence of the different clones was confirmed by sequencing of both DNA strands with the pUCM13 standard primers. The Acc. Nos for VP4 and/or VP7 sequences reported in this study are: SONMX32 (KP119460, KP119464,), SONMX-37 (KP119461, KP119465), SONMX-38 (KT852964), SONMX-39 (KT852962), SONMX-51 (KT852965), SONMX-62 (KT852963), SONMX-99 (KP119462, KP119466), SONMX-124 (KP119463, KP119467).
Phylogenetic analysis and sequence alignment. The sequences were analyzed using MEGA 5.0 software (Tamura et al., 2011) and compared with other sequences reported in the GenBank database, including the VP4 and VP7 gene sequences of the licensed vaccines Rotarix and RotaTeq. The phylogenetic relationships between strains were reconstructed using the maximum likelihood method with 1000 replicates to test phylogeny (Tamura et al., 2011). The phylogenetic analysis was achieved using the partial G12 gene sequence (nt 87-1016) and the $\mathrm{P}[8]$ sequence (nt 142-762). The deduced amino acid (aa) sequence alignment of VP7 and VP4 was achieved using the partial sequence of the antigenic region of VP7 (aa 80-175) and VP8* (aa 45-233). The NCBI Acc. Nos for the sequences for VP7 analysis are: KDH651 (AB861961), ISO125 (EU016497), ISO120 (EU016451), 05SLC009 (AB306268), 04S027(AB263987), Dhaka25(DQ146654), Se585/USA(AJ311741), RV176 (DQ490556),B4633(DQ146643), ISO1212(EU016460), MD844 (AB269689), US6597 (FJ152121), CAU 195 (EF059916), CAU 214(EF059917), MatLab13(DQ146676), Dhaka12(DQ146665), MS310-06(AB436814), 3133WC(HQ657154), KDH684(AB861972), KDH633(AB861950), CP727 (AB125852), T152 (AB071404), HC91 (AY855065), Arg720A (EU496257), ARGENTINA (DQ111868) and L26 (M58290). The NCBI Acc. Nos of the sequences VP4 (region VP8*) analysis are: KDH651(AB861959), DPRU75 (KJ752364), DPRU4620 (KJ753730), BTN-120 (AB905370), HR15 (KJ855216), Ro4439 (JN711100), RT095-07 (JQ069655.1), UK-HLD (JX411972), VU08-09-40 (KC442932), VU08-09-39 (JF790352), R1949 (HM035525), 1670SR (KJ412735), Arg7500 (JN088445), M12-15 (KC484719), GRAVP430 (AB553325), 8340 (JN580414), BE00097(JN258920), STHY125(JX470501), MX07623 (JX012339), MX07-588 (JX012338), MX07-577 (JX012337),

\begin{tabular}{|c|c|c|c|c|c|c|c|c|c|c|c|c|c|c|c|c|c|c|c|c|c|c|c|c|}
\hline Strain & G/Lineage & & & & & $7-1 a$ & & & & & & & $7-1$ & $1 \mathrm{~b}$ & & & & & & $7-2$ & & & & \\
\hline Rotarix & G1 & $s \frac{87}{T}$ & $\stackrel{91}{\mathrm{~T}} \stackrel{94}{\mathrm{~N}}$ & $\begin{array}{l}96 \\
\mathrm{G}\end{array}$ & $\stackrel{97}{E}$ & $\begin{array}{l}104 \\
Q\end{array}$ & $\begin{array}{c}123 \\
\mathrm{~S}\end{array}$ & $\sqrt[125]{V}$ & $\sqrt[129]{V}$ & $\begin{array}{l}130 \\
D\end{array}$ & $\begin{array}{l}291 \\
\mathrm{~K}\end{array}$ & $\begin{array}{c}201 \\
Q\end{array}$ & $\stackrel{211}{N}$ & $\mathrm{~N}^{238}$ & $\stackrel{242}{T}$ & $\begin{array}{l}143 \\
K\end{array}$ & $\begin{array}{c}145 \\
D\end{array}$ & $\begin{array}{c}146 \\
Q\end{array}$ & $\stackrel{147}{N}$ & $\stackrel{148}{\mathrm{~L}}$ & $\begin{array}{cc}190 & 217 \\
S & M\end{array}$ & $17 \frac{2 \pi}{N}$ & $\begin{array}{c}21 \\
\mathrm{~N}\end{array}$ & \\
\hline RotaTeq & G1 & . & - . & & $\mathrm{D}$ & . & - & - & - & 政 & . & & & & & & & 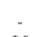 & $\mathrm{S}$ & & 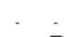 & & . & \\
\hline L26 & $\mathrm{G} 12 / \mathrm{I}$ & $\mathrm{N}$ & $\mathrm{T}$ & $\mathrm{P}$ & D & . & D & A & & $\mathbf{N}$ & . & . & $\mathrm{D}$ & . & N & $Q$ & $Q$ & $\mathrm{~N}$ & S & 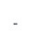 & $\mathrm{E}$ & A & A & \\
\hline $\mathrm{Arg} 720 \mathrm{~A}$ & G12/II & s & . $T$ & $\mathrm{P}$ & $\mathrm{D}$ & . & D & A & . & - & . & . & $\mathrm{D}$ & . & N & $Q$ & $Q$ & $\mathrm{~N}$ & S & 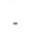 & $E$ & A & A & \\
\hline HC91 & $\mathrm{G} 12 / \mathrm{II}$ & $\mathrm{s}$ & . $\mathrm{T}$ & $\mathrm{P}$ & D & . & D & A & . & . & . & . & D & . & $\mathrm{N}$ & $Q$ & $Q$ & $N$ & $\mathrm{~S}$ & . & . $E$ & A & A & \\
\hline T152 & G12/II & $\mathrm{s}$ & - $\mathrm{T}$ & $\mathrm{P}$ & D & . & D & A & . & . & . & . & D & . & N & $Q$ & $Q$ & $\mathrm{~N}$ & $\mathrm{~S}$ & . & $\mathrm{E}$ & A & A & \\
\hline CP727 & G12/II & $\mathrm{s}$ & $\therefore \mathrm{T}$ & $P$ & D & . & D & A & & . & . & . & D & . & $\mathrm{N}$ & $\mathrm{Q}$ & $Q$ & $\mathrm{~N}$ & $\mathrm{~S}$ & . & $\mathrm{E}$ & A & A & \\
\hline KDH651 & G12/II & $S$ & . $\mathrm{T}$ & $\mathrm{P}$ & D & . & D & $\mathrm{S}$ & . & . & . & . & D & . & $\mathrm{N}$ & $Q$ & $Q$ & $\mathrm{~N}$ & S & . & $\mathrm{E}$ & E & A & \\
\hline ISO125 & G12/III & $\mathrm{S}$ & . $\mathrm{T}$ & $\mathrm{P}$ & $\mathrm{D}$ & . & D & $\mathrm{S}$ & . & . & 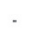 & . & $\mathrm{D}$ & . & N & $Q$ & $Q$ & $\mathrm{~N}$ & S & . & $E$ & A & A & \\
\hline 05SLC009 & G12/II & $\mathrm{S}$ & $\mathrm{T}$ & $\mathrm{P}$ & D & . & D & $\mathrm{S}$ & . & . & 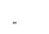 & . & $\mathrm{D}$ & . & N & $Q$ & $Q$ & $\mathrm{~N}$ & $S$ & . & $E$ & $E$ & A & \\
\hline $04 S 027$ & G12/II & $\mathrm{S}$ & $\mathrm{T}$ & $\mathrm{P}$ & $\mathrm{D}$ & . & D & $\mathrm{S}$ & . & . & 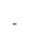 & & $\mathrm{D}$ & . & $\mathbb{N}$ & $Q$ & $Q$ & $\mathrm{~N}$ & S & . & $E$ & A & A & \\
\hline Dhaka25 & G12лIII & $\mathrm{s}$ & $\mathrm{T}$ & $\mathrm{P}$ & D & . & D & $\mathrm{S}$ & . & . & & & D & . & $\mathrm{N}$ & $\mathrm{Q}$ & $Q$ & $\mathrm{~N}$ & $\mathrm{~S}$ & 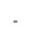 & $\mathrm{E}$ & A & A & \\
\hline B4633 & G12/III & $\mathrm{s}$ & $\mathrm{T}$ & $\mathrm{P}$ & D & . & D & $\mathrm{S}$ & . & . & & & D & & $\mathrm{N}$ & $Q$ & $Q$ & $\mathrm{~N}$ & $\mathrm{~S}$ & & $E$ & E & A & \\
\hline Se585 & G12/III & $\mathrm{s}$ & . $T$ & $\mathrm{P}$ & D & . & D & $\mathrm{S}$ & . & . & 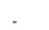 & & D & . & N & $Q$ & $Q$ & $\mathrm{~N}$ & S & . & . $E$ & $E A$ & A & \\
\hline $3133 \mathrm{WC}$ & G12JII & $S$ & $\mathrm{~T}$ & $\mathrm{P}$ & D & . & D & $\mathrm{S}$ & . & . & . & . & $\mathrm{D}$ & . & N & $Q$ & $Q$ & $\mathrm{~N}$ & S & . & $E$ & A & A & \\
\hline SONMX-32 & G12/III & $\mathrm{s}$ & $\mathrm{T}$ & $\mathrm{P}$ & D & . & D & $\mathrm{S}$ & . & . & . & . & $\mathrm{D}$ & $N$ & N & $Q$ & $Q$ & $\mathrm{~N}$ & $\mathrm{~S}$ & & E & E & A & \\
\hline SONMX-37 & G12JIII & $\mathrm{s}$ & $\mathrm{T}$ & $\mathrm{P}$ & D & I & D & $\mathrm{S}$ & . & - & - & & D & N & N & Q & $Q$ & $\mathrm{~N}$ & $\mathrm{~S}$ & & $E$ & E & A & \\
\hline SONMX-39 & $\mathrm{G} 12 / \mathrm{II}$ & S & $\mathrm{T}$ & $\mathrm{P}$ & D & . & D & $\mathrm{S}$ & . & . & & & $\mathrm{D}$ & N & $\mathbb{N}$ & $Q$ & $Q$ & $\mathrm{~N}$ & $S$ & & $E$ & E & A & \\
\hline SONMX-51 & $\mathrm{G} 12 \pi \mathrm{II}$ & $S$ & $\mathrm{~T}$ & $\mathrm{P}$ & D & & D & $\mathrm{S}$ & . & . & & & D & & $N$ & $Q$ & $Q$ & $\mathrm{~N}$ & S & & $E$ & E & A & \\
\hline SONMX-99 & G12/III & S & $\mathrm{T}$ & $\mathrm{P}$ & D & . & D & $\mathrm{S}$ & . & . & & & D & & $\mathrm{N}$ & $Q$ & $Q$ & $\mathrm{~N}$ & S & & $\mathrm{E}$ & E & A & \\
\hline SONMX-124 & G12/III & $\mathrm{s}$ & $\mathrm{T}$ & $\mathrm{P}$ & D & [ & D & $\mathrm{S}$ & . & . & & & D & & $\mathrm{N}$ & $Q$ & $Q$ & $\mathrm{~N}$ & $\mathrm{~s}$ & & $\mathrm{E}$ & E & A & \\
\hline DQ204743 & G12/lV & $\mathrm{N}$ & $T$ & $\mathrm{P}$ & & & D & A & & & & & D & & $\mathrm{N}$ & $Q$ & $Q$ & $\mathrm{~N}$ & $S$ & & & E A & A & \\
\hline
\end{tabular}

Fig. 2

Amino acid sequence analysis of the VP7 gene of genotype G12 rotavirus

Alignment of antigenic residues of the VP7 region from genotype G12 rotavirus strains. 


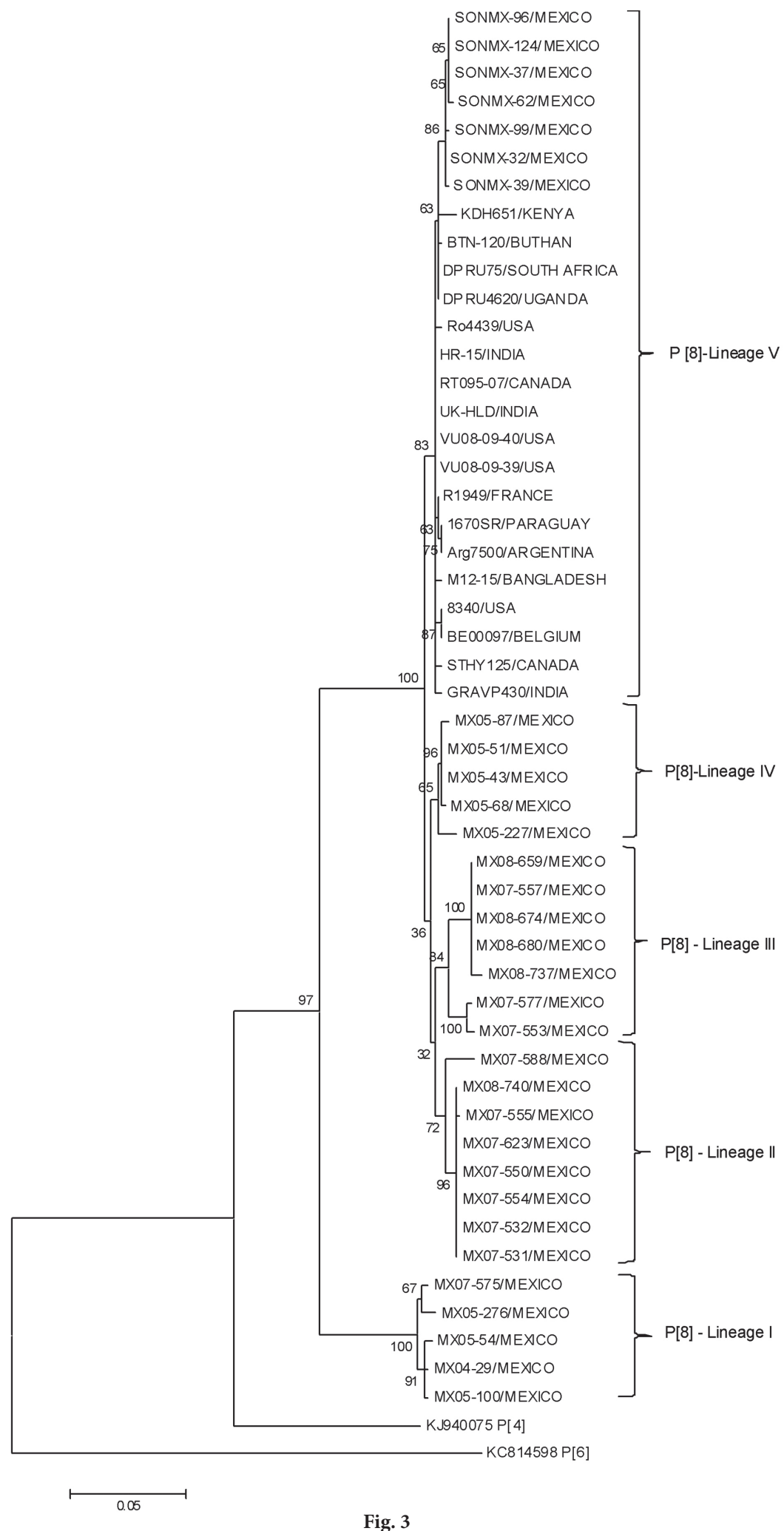

Fig. 3

Phylogenetic analysis of the VP4 gene of genotype $P[8]$ rotavirus

Phylogenetic analysis of the nucleotide sequence of the VP4 gene from genotype P[8] rotavirus strains reported in this study (SONMX-32, SONMX-37, SONMX-39, SONMX-62, SONMX-99, SONMX-124) along with reference strains downloaded from the GenBank database. The statistical significance of the phylogenetic trees constructed was estimated by the bootstrap method (1000 replicates). 
MX07-575 (JX012336),MX07-553 (JX012335), MX07-550 (JX012334), MX05-276 (JX012333), MX05-227 (JX012332), MX05100 (JX012331), MX05-54 (JX012330), MX08-737 (HQ585866), MX08-680 (HQ585865), MX08-674 (HQ585864), MX08-740 (FJ665391), MX08-659 (FJ665390), MX07-557 (FJ665389), MX07555 (FJ665388), MX07-554 (FJ665387), MX07-532 (FJ665386), MX07-531 (FJ665385), MX05-68 (FJ665384), MX05-87 (FJ665383), MX05-51 (FJ665382), MX05-43 (FJ665381), MX04-29 (FJ665380), SC0028_P[6](KC814598) and KJ940075(SC19868_P[4]).

\section{Results}

\section{Rotavirus detection and genotyping}

Fourteen of the 100 samples analyzed were positive for rotavirus. All positive samples were genotyped by RT-PCR amplification of the VP4 gene (P genotype) and the VP7 gene (G genotype) (Gouvea et al., 1990; Gentsch et al., 1992; Das et al., 1994; Simmonds et al., 2008). Successful amplification was achieved for VP4 in 7/14 (50\%) of the rotavirus positive samples and for VP7 in 6/14 (43\%) of samples. Of the G genotypes, G12P[8] was detected in 4/14 (28.5\%), G12P (not typed) was detected in $2 / 14(14.3 \%)$, and $\mathrm{G}$ (not typed) $\mathrm{P}[8]$ was detected in $3 / 14(21.42 \%$ ) rotavirus positive samples.

\section{Phylogenetic analysis and sequence alignment}

Nucleotide sequence analysis of the VP7 gene of the G12 genotype reported in this study, revealed 98-99\% identity with other G12 rotavirus strains, previously reported in Kenya (AB861961), India (EU016453), and Sri Lanka (AB306268) (Komoto et al., 2014; Paul et al., 2010). Phylogenetic analysis of the VP7 gene showed that G12 genotypes clustered into lineage III (Fig. 1). The alignment of antigenic residues of VP7 genotype G12 revealed some amino acid variations with respect to genotype G1 of Rotarix and RotaTeq (Fig. 2)

Moreover, the sequence of the VP8* region in the VP4 protein of rotavirus detected in this study shared 99\% identity with rotavirus strains from Kenya (AB861959), Uganda (KJ753730), South Africa (KJ752364), and Bhutan (AB905370). The phylogenetic tree of the VP4 sequences revealed different lineages of $\mathrm{P}[8]$. The gene sequences of $\mathrm{P}[8]$ reported in this study, which were isolated in 2013, belonged to lineage $\mathrm{V}$, whereas other sequences reported previously in México, isolated in 2005, 2007, and 2008, clustered in different lineages (Fig. 3). Furthermore, the deduced amino acid sequence of the antigenic region of VP8* revealed the presence of sequence variations in some known antigenic epitopes in comparison with the rotavirus $\mathrm{P}[8]$ genotype of the vaccine strains Rotarix ${ }^{\circledast}$ and RotaTeq ${ }^{\oplus}$ (Fig. 4).

\begin{tabular}{|c|c|c|c|c|c|c|c|c|c|c|c|c|c|c|c|c|c|c|c|c|c|c|c|c|c|}
\hline \multirow{2}{*}{$\begin{array}{l}\text { Rotavirus } \\
\text { Strains }\end{array}$} & \multicolumn{11}{|c|}{ WP8-1 } & \multicolumn{2}{|c|}{ VPP-2 } & \multicolumn{9}{|c|}{ WPB-3 } & \multicolumn{3}{|c|}{ VP8-4 } \\
\hline & 100 & 145 & 147 & 149 & 187 & 189 & 191 & 192 & 193 & 194 & 195 & 179 & 182 & 113 & 114 & 115 & 116 & 125 & 131 & 132 & $13: 3$ & 135 & & 88 & 89 \\
\hline ROTARIX G1P[8] & $\mathrm{D}$ & $\mathrm{s}$ & $\mathrm{s}$ & $\mathrm{N}$ & $\mathrm{s}$ & $\mathrm{s}$ & A & $\mathrm{N}$ & $\mathrm{L}$ & $\mathrm{N}$ & $\mathrm{N}$ & $E$ & $\mathrm{R}$ & $\mathrm{N}$ & $P$ & $\mathrm{~V}$ & $\mathrm{D}$ & $\mathrm{s}$ & $\mathrm{s}$ & $\mathrm{N}$ & $\mathrm{D}$ & $\mathbf{N}$ & $\mathrm{N}$ & $T$ & $\bar{N}$ \\
\hline ROTATEQ G6P[8] & & . & & & & $\mathrm{N}$ & . & & . & . & D & . & & & & & . & $\mathrm{N}$ & $\mathbf{R}$ & & & D & & . & \\
\hline MX04-29 & . & . & . & . & . & . & . & . & . & . & . & . & . & T & . & . & . & . & . & . & . & . & . & . & . \\
\hline MX05-43 & . & G & . & . & & $\mathrm{N}$ & & . & . & . & $\mathrm{G}$ & . & . & . & . & . & . & $\mathrm{N}$ & $\mathrm{R}$ & . & . & D & . & . & . \\
\hline MX05-51 & . & G & . & . & & $\mathrm{N}$ & & . & & . & G & & . & & & . & & $\mathrm{N}$ & $\mathbf{R}$ & . & & D & . & . & . \\
\hline MX05-54 & & . & & . & & 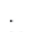 & & & & . & & . & . & T & & & . & & 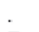 & & E & & . & . & . \\
\hline MX05-68 & & G & & . & . & $\mathrm{N}$ & . & & . & . & G & . & . & . & & . & . & $\mathrm{N}$ & $\mathrm{R}$ & & & D & . & . & . \\
\hline MX05-87 & . & G & . & . & . & $\mathrm{N}$ & . & . & . & . & G & . & . & . & . & . & . & $\mathrm{N}$ & $\mathrm{R}$ & . & . & D & . & . & . \\
\hline MX05-227 & . & G & . & . & & $\mathrm{N}$ & & . & . & . & G & . & . & & . & . & . & $\mathrm{N}$ & $\mathrm{R}$ & . & . & D & . & . & . \\
\hline MX05-276 & & & & . & & & & & & & & & & $\mathrm{T}$ & & & & & & & & & & . & \\
\hline MX07-531 & . & G & . & . & & $\mathrm{N}$ & & . & . & . & G & . & . & D & . & . & $\mathrm{N}$ & $\mathrm{N}$ & $\mathrm{R}$ & . & . & D & . & . & . \\
\hline MX07-532 & . & G & . & . & . & $\mathrm{N}$ & . & . & . & . & G & . & . & D & . & . & $\mathrm{N}$ & $\mathrm{N}$ & $\mathrm{R}$ & . & . & D & . & . & . \\
\hline MX07-550 & & G & & . & . & $\mathrm{N}$ & . & & . & . & G & . & & D & & & $\mathrm{N}$ & $\mathrm{N}$ & $\mathrm{R}$ & & & D & & . & . \\
\hline MX07-553 & . & $\mathrm{G}$ & . & . & & $\mathrm{N}$ & & . & & . & G & & . & D & & . & . & $\mathrm{N}$ & $\mathrm{R}$ & . & . & D & . & . & . \\
\hline MX07-554 & . & $\mathrm{G}$ & . & . & & $\mathrm{N}$ & & & & . & G & & . & D & & . & $\mathrm{N}$ & $\mathrm{N}$ & $\mathrm{R}$ & & & D & . & . & . \\
\hline MX07-555 & . & G & . & . & & $\mathrm{N}$ & & . & . & . & G & . & . & D & . & . & $\mathrm{N}$ & $\mathrm{N}$ & $\mathrm{R}$ & . & . & D & . & . & . \\
\hline MX07-557 & . & G & . & . & . & $\mathrm{N}$ & . & . & . & . & G & . & . & D & . & . & . & $\mathrm{N}$ & $\mathrm{R}$ & . & . & D & . & . & . \\
\hline MX07-575 & & . & & & & & & & . & . & & . & & T & & & . & & . & & & . & & . & . \\
\hline MX07-577 & . & G & & . & . & $\mathrm{N}$ & . & & . & . & $\mathrm{G}$ & . & . & D & & & . & $\mathrm{N}$ & $\mathrm{R}$ & & & D & . & . & . \\
\hline MX07-588 & . & G & . & . & & $\mathrm{N}$ & & . & . & . & G & . & . & D & . & . & . & $\mathrm{N}$ & $\mathrm{R}$ & . & . & D & . & . & . \\
\hline MX07-623 & . & G & . & . & & $\mathrm{N}$ & & . & & . & G & & . & D & & . & $\mathrm{N}$ & $\mathrm{N}$ & $\mathrm{R}$ & & & D & . & . & . \\
\hline MX08-659 & . & G & . & . & . & $\mathrm{N}$ & . & . & . & . & G & . & . & D & . & . & . & $\mathrm{N}$ & $\mathrm{R}$ & . & . & D & . & . & . \\
\hline MX08-674 & & G & & & & $\mathrm{N}$ & & & . & . & G & . & & D & & & . & $\mathrm{N}$ & $\mathrm{R}$ & & & D & & . & . \\
\hline MX08-680 & . & G & . & . & & $\mathrm{N}$ & & . & . & . & G & . & . & D & & . & . & $\mathrm{N}$ & $\mathrm{R}$ & . & & D & . & . & . \\
\hline MX08-737 & . & G & $\mathrm{N}$ & . & & $\mathrm{N}$ & & . & . & . & G & . & . & D & . & . & . & $\mathrm{N}$ & $\mathrm{R}$ & . & . & D & . & . & . \\
\hline MX08-740 & . & G & . & & & $\mathrm{N}$ & & . & . & . & G & . & . & D & . & . & $\mathrm{N}$ & $\mathrm{N}$ & $\mathrm{R}$ & . & . & D & . & . & . \\
\hline SONMX-32 & . & G & . & T & & $\mathrm{N}$ & & . & . & . & G & . & . & . & . & . & . & $\mathrm{N}$ & $\mathrm{R}$ & . & . & D & . & . & . \\
\hline SONMX-37 & . & G & . & T & . & $\mathrm{N}$ & . & . & . & . & G & . & . & . & . & . & . & $\mathrm{N}$ & $\mathrm{R}$ & . & . & D & . & . & . \\
\hline SONMX-39 & . & G & & T & . & $\mathrm{N}$ & . & . & . & . & G & . & . & . & & . & . & $\mathrm{N}$ & $\mathrm{R}$ & & & D & . & . & . \\
\hline SONMX-62 & . & G & . & T & & $\mathrm{N}$ & & . & . & . & G & . & . & . & . & . & . & $\mathrm{N}$ & $\mathrm{R}$ & . & . & D & . & . & . \\
\hline SONMX-99 & . & G & . & T & & $\mathrm{N}$ & & . & . & . & G & & . & & & . & & $\mathrm{N}$ & $\mathrm{R}$ & . & . & D & . & . & . \\
\hline SONMX-124 & & $\mathrm{G}$ & & T & & $\mathrm{N}$ & & & & & G & & & & & & & $\mathrm{N}$ & $\mathrm{R}$ & & & D & & & \\
\hline
\end{tabular}

Fig. 4 


\section{Discussion}

Our results demonstrated the presence of rotavirus G12P [8] in México. To our knowledge, this is the first report of this emerging rotavirus strain in this country. Following the first report of G12 in 1987, this genotype was not reported again until 1998 (Rahman et al., 2007). Over the past few years this genotype has been reported as an uncommon but emerging genotype in Asia, Europe, South America, Africa, and North America (Rahman et al., 2007; Ray et al., 2007; WHO, 2011; da Silva Soares et al., 2012; Komoto et al., 2014). Based on the increasing number of countries reporting human $\mathrm{G} 12$ rotavirus infection, it is possible that this rotavirus genotype will become globally dominant in the future (Patton, 2012). Phylogenetic analysis of G12 and P[8] showed an association with G12-lineage III and P[8]-lineage V. The amino acid variations in the antigenic residues of VP7 and VP4 may be indicative of strains evolving from the licensed rotavirus vaccines Rotarix ${ }^{\circledast}$ and RotaTeq $q^{\oplus}$; however, more studies are required to investigate this hypothesis. This and other studies have reported the changing profile of rotavirus genotypes, with the detection of emerging and uncommon genotypes before and after the rotavirus vaccine program was introduced (Banerjee et al., 2007; Le et al., 2008; Bányai et al., 2012; Patton, 2012; Afrad et al., 2013; Wangchuk et al., 2014). Although there is evidence that the implementation of rotavirus vaccines has reduced rotavirus gastroenteritis severity and associated hospitalizations (Cortes et al., 2011; Leshem et al., 2014), the constantly evolving genotype diversity may represent a challenge to the efficacy of currently available vaccines. Therefore, worldwide surveillance of circulating rotavirus genotypes is crucial to monitor the effectiveness of current rotavirus vaccines and to direct the development of novel vaccines in the future.

Acknowledgements. The authors thank Dr. Ricardo Gómez-Flores, Universidad Autónoma de Nuevo León, Facultad de Ciencias Biológicas, Laboratorio de Inmunología y Virología, San Nicolás de los Garza, Nuevo León, México, for helpful contributions regarding English language editing. This study was supported by PRODEP (DSA/103.5/14/11117).

\section{References}

Afrad MH, Hassan Z, Farjana S, Moni S, Barua S, Das SK, Faruque AS, Azim T, Rahman M (2013): Changing profile of rotavirus genotypes in Bangladesh, 2006-2012. BMC. Infect. Dis. 13, 320. http://dx.doi.org/10.1186/14712334-13-320

Banerjee I, Ramani S, Primrose B, Iturriza-Gomara M, Gray JJ, Brown DW, Kang G (2007): Modification of rotavirus multiplex RT-PCR for the detection of G12 strains based on characterization of emerging G12 rotavirus strains from South India. J. Med Virol. 79, 1413-1421. http:// dx.doi.org/10.1002/jmv.20872

Bányai K, László B, Duque J, Steele AD, Nelson EAS, Gentsch JR, Parashar UD (2012): Systematic review of regional and temporal trends in global rotavirus strain diversity in the pre rotavirus vaccine era: insights for understanding the impact of rotavirus vaccination programs. Vaccine 30, A122-A130. http://dx.doi.org/10.1016/j.vaccine.2011.09.111

Cortes JE, Curns AT, Tate JE, Cortese MM, Patel MM, Zhou F, Parashar UD (2011): Rotavirus vaccine and health care utilization for diarrhea in US children. N. Engl. J. Med. 365, 1108-1117. http://dx.doi.org/10.1056/NEJMoa1000446

da Silva Soares L, dos Santos Lobo P, Mascarenhas JDAP, Neri DL, dos Santos Guerra SdF, de Oliveira AdSL, Maestri RP, de Souza Oliveira D, da Costa Linhares A (2012): Identification of lineage III of G12 rotavirus strains in diarrheic children in the Northern Region of Brazil between 2008 and 2010. Arch. Virol. 157, 135-139. http:// dx.doi.org/10.1007/s00705-011-1111-Z

Das BK, Gentsch JR, Cicirello HG, Woods PA, Gupta A, Ramachandran M, Kumar R, Bhan M, Glass RI (1994): Characterization of rotavirus strains from newborns in New Delhi, India. J. Clin. Microbiol. 32, 1820-1822.

Delogu R, Ianiro G, Camilloni B, Fiore L, Ruggeri FM (2015): Unexpected spreading of $\mathrm{G} 12 \mathrm{P}[8]$ rotavirus strains among young children in a small area of central Italy. J. Med. Virol. 87,1292-1302. http://dx.doi.org/10.1002/jmv.24180

Desselberger U, Manktelow E, Li W, Cheung W, Iturriza-Gómara M, Gray J (2009): Rotaviruses and rotavirus vaccines. Br. Med. Bull. 90, 37-51. http://dx.doi.org/10.1093/ bmb/ldp009

Gentsch J, Glass R, Woods P, Gouvea V, Gorziglia M, Flores J, Das B, Bhan M (1992): Identification of group A rotavirus gene 4 types by polymerase chain reaction. J. Clin Microbiol. 30, 1365-1373.

Gouvea V, Glass RI, Woods P, Taniguchi K, Clark HF, Forrester B, Fang Z (1990): Polymerase chain reaction amplification and typing of rotavirus nucleic acid from stool specimens. J. Clin. Microbiol. 28, 276-282.

Komoto S, Apondi EW, Shah M, Odoyo E, Nyangao J, Tomita M, Wakuda M, Maeno Y, Shirato H, Tsuji T (2014): Whole genomic analysis of human G12P [6] and G12P [8] rotavirus strains that have emerged in Kenya: Identification of porcine-like NSP4 genes. Infect. Genet. Evol. 27, 277-293. http://dx.doi.org/10.1016/j.meegid.2014.08.002

Le VP, Kim JY, Cho SL, Nam SW, Lim I, Lee HJ, Kim K, Chung SI, Song W, Lee KM (2008): Detection of unusual rotavirus genotypes G8P [8] and G12P [6] in South Korea. J. Med. Virol. 80, 175-182. http://dx.doi.org/10.1002/jmv.21044

Leshem E, Moritz RE, Curns AT, Zhou F, Tate JE, Lopman BA, Parashar UD (2014): Rotavirus vaccines and health care utilization for diarrhea in the United States (2007-2011). Pediatrics 134, 15-23. http://dx.doi.org/10.1542/peds.2013-3849

Parashar U, Steele D, Neuzil K, Quadros Cd, Tharmaphornpilas P, Serhan F, Santosham M, Patel M, Glass R (2013): Progress with Rotavirus Vaccines: Summary of the Tenth International Rotavirus Symposium. 
Patton JT (2012): Rotavirus diversity and evolution in the postvaccine world. Discov Med. 13, 85.

Paul S, Ahmed M, Hossain M, Mahmud M, Bhuiyan M, Saha S, Tabassum S (2010): Molecular characterization of group A human rotavirus among hospitalized children and adults in Bangladesh: finding of emerging G12 strain. Mymensingh Med. J. 19, 16-26.

Rahman M, Matthijnssens J, Yang X, Delbeke T, Arijs I, Taniguchi K, Iturriza-Gómara M, Iftekharuddin N, Azim T, Van Ranst M (2007): Evolutionary history and global spread of the emerging G12 human rotaviruses. J. Virol. 81, 2382-2390. http://dx.doi.org/10.1128/JVI.01622-06

Ray P, Sharma S, Agarwal R, Longmei K, Gentsch J, Paul V, Glass $R$, Bhan M (2007): First detection of G12 rotaviruses in newborns with neonatal rotavirus infection at all India Institute of Medical Sciences, New Delhi, India. J. Clin. Microbiol. 45, 3824-3827. http://dx.doi.org/10.1128/ JCM.01288-07

Santos N, Hoshino Y (2005): Global distribution of rotavirus serotypes/genotypes and its implication for the development and implementation of an effective rotavirus vaccine. Rev. Med. Virol. 15, 29-56. http://dx.doi.org/10.1002/ $\underline{\operatorname{rmv} .448}$

Simmonds MK, Armah G, Asmah R, Banerjee I, Damanka S, Esona M, Gentsch JR, Gray JJ, Kirkwood C, Page N (2008): New oligonucleotide primers for P-typing of rotavirus strains: Strategies for typing previously untypeable strains. J. Clin. Virol. 42, 368-373. http://dx.doi.org/10.1016/j. jcv.2008.02.011
Tamura K, Peterson D, Peterson N, Stecher G, Nei M, Kumar S (2011): MEGA5: molecular evolutionary genetics analysis using maximum likelihood, evolutionary distance, and maximum parsimony methods. Mol. Biol. Evol. 28, 2731-2739. http://dx.doi.org/10.1093/ molbev/msr121

Tate JE, Burton AH, Boschi-Pinto C, Steele AD, Duque J, Parashar UD (2012): 2008 estimate of worldwide rotavirusassociated mortality in children younger than 5 years before the introduction of universal rotavirus vaccination programmes: a systematic review and meta-analysis. Lancet Infect. Dis. 12, 136-141. http://dx.doi.org/10.1016/ S1473-3099(11)70253-5

Tort LF, Victoria M, Lizasoain AA, Castells M, Maya L, Gomez MM, Arreseigor E, Lopez P, Cristina J, Leite JP, Colina R (2015): Molecular epidemiology of group a rotavirus among children admitted to hospital in Salto, Uruguay, 2011-2012: First detection of the emerging genotype G12. J. Med. Virol. 87, 754-763. http://dx.doi.org/10.1002/jmv.24123

Wangchuk S, Mitui MT, Tshering K, Yahiro T, Bandhari P, Zangmo S, Dorji T, Tshering K, Matsumoto T, Nishizono A (2014): Dominance of Emerging G9 and G12 Genotypes and Polymorphism of VP7 and VP4 of Rotaviruses from Bhutanese Children with Severe Diarrhea Prior to the Introduction of Vaccine. PloS one 9, e110795. http:// dx.doi.org/10.1371/journal.pone.0110795

WHO (2011): Global rotavirus information and surveillance bulletin. Reporting period: January through December 2010. World Health Organization 4. 